\title{
Indicadores propuestos para medir el impacto de la Responsabilidad Social Universitaria
}

\author{
Martha Elena López Regalado* \\ Juan Manuel Perusquia Velasco** \\ Rocío Villalón CaÑas*** \\ México
}

\section{Resumen}

El estudio que se presenta tiene el propósito de dar a conocer la importancia de la Responsabilidad Social Universitaria (RSU) para la sociedad que demanda un mejor desempeño, cuya gestión garantice la construcción del conocimiento con comportamiento ético y socialmente responsable, que se evalué en forma constante a través de indicadores.

\section{Introducción}

En los últimos años el desarrollo que ha experimentado la responsabilidad social; ha sido fundamental en cualquier tipo de organización,

* Magíster en Administración General. Universidad Autónoma de Baja California. Coordinación de Costos. Contacto: marthalopez@uabc.edu.mx

* Dr. en Administración. Universidad Autónoma de Baja California. Coordinación de Movilidad Académica. Contacto: perusquia@uabc.edu.mx

**:Master in Business Administration. Universidad Autónoma de Baja California. Coordinación de Prácticas Profesionales. Contacto: rocio.villalon@ uabc.edu.mx 
ya sea pública, privadas con o sin fines de lucro, sin dejar de incluir las Instituciones de Educación Superior (IES) como parte esencial del desarrollo. Hablar de la responsabilidad social es centrar la atención en la gestión y realización de prácticas sociales dentro de la universidad que agreguen un valor estratégico bajo el mismo concepto de responsabilidad social ante sus diferentes grupos de interés (stakeholders) con los que interactúa.

Las IES se encuentran inmersas como factor clave en el desarrollo del país en el ámbito de la responsabilidad social, representando para ellas un nuevo reto encaminado a la calidad y la pertinencia que busca dar respuesta a las demandas del desarrollo económico, político y social del país.

Para lograrlo se requiere una apropiada articulación entre sus funciones de docencia, gestión, investigación y extensión, que fortalezcan sus vínculos con los diferentes sectores productivos, la comunidad y la sociedad en general dentro de un entorno globalizado. En las últimas décadas se han logrado grandes avances en cuestión de responsabilidad social, uno de ellos ha sido la transición que ha tenido el concepto, pasando de un enfoque filantrópico a otro que pretende desarrollar una toma de conciencia global, compleja y holística de la organización, centrada en el impacto causado por su actividad (Vallaeys, 2006). Sin embargo, pese a que se ha convertido en una necesidad para el bien común, se puede plantear que son pocas las universidades que se han comprometido realmente con proyectos de responsabilidad social y el desarrollo sustentable con impacto en la comunidad.

\section{Metodología}

El presente trabajo se deriva de una investigación de tipo exploratoria, descriptiva y propositiva que tiene como objetivo mostrar los diversos métodos e indicadores expuestos por diferentes autores, utilizados en algunas universidades, para desarrollar un instrumento propio que ayude a realizar el diagnóstico de la Responsabilidad Social Universitaria (RSU), en la Facultad de Contaduría y Administración de la Universidad Autónoma de Baja California, a fin de medir el impacto de 
la misma y establecer estrategias que orienten las acciones y prácticas hacia la competitividad y desarrollo del país, mediante la formación integral de los futuros profesionistas que darán respuesta a la problemática actual, en la construcción de una sociedad justa, solidaria y equitativa socialmente.

Con base en los diferentes instrumentos encontrados y las aportaciones realizadas por el Banco Interamericano de Desarrollo (BID), el Proyecto Universidad Construye País de Chile y la Asociación Ausjal. Al medir la responsabilidad social se pretende lograr un desarrollo sostenible, sustentado en el compromiso que asume la organización con sus stakeholders para dar cumplimiento a las obligaciones y necesidades económicas, sociales y medioambientales. Con base en los resultados obtenidos, se proponen estrategias que solucionen los problemas del entorno con un comportamiento ético en la gestión y desarrollo de productos y servicios (Valarezo y Túñez, 2014).

La sociedad demanda que las universidades desempeñen efectivamente su rol como instituciones responsables, incorporando el tema de RSU en sus procesos de gestión que garanticen la construcción del conocimiento de vanguardia con comportamiento ético y socialmente responsable en todas las funciones y áreas de la institución. La responsabilidad social universitaria es en sí una estrategia integrada de gestión que se puede medir y controlar en su desarrollo económico, social y medioambiental.

\section{Antecedentes de la Responsabilidad Social Universitaria}

Es necesario hablar sobre la Responsabilidad Social (RS) por varias razones, una es que se le considera como parte la estética que repercute en la imagen de la organización, desvirtuando su importancia y debilitando la claridad en la aplicación del concepto. La RS va más allá de ser un instrumento estratégico, más bien es considerado como un distintivo de calidad, que busca la transformación social en el desarrollo, y en el crecimiento económico (Hernández y Saldarriaga, 2009). 
La sociedad cada día demanda una mayor participación por parte de la academia y universidad en hechos sociales (Vallaeys, 2006). La participación de toda la comunidad universitaria se debe dar en el marco de un ejercicio responsable, de respeto a la libertad, la ética y la tolerancia con una perspectiva de compromiso y pertinencia institucional (Martínez C., 2011).

Las universidad como institución que construye y genera conocimiento es responsable de la formación de profesionistas en el ámbito cultural, social y medioambiental, fortaleciendo los vínculos con los diferentes sectores productivos, el gobierno y la sociedad en general, dando respuesta a las necesidades y problemas que aquejan a la comunidad y para enfrentar los nuevos retos del país (Vázquez, et al., 2006).

La RS nace en las empresas, sin embargo en las últimas décadas se presenta como política de gestión en el campo universitario en respuesta a los impactos organizacionales y académicos que viven las IEs, algunas se ya se encuentran inmersas en actividades relacionadas con el cuidado del medioambiente y/o con el bien común (Domínguez, 2009).

Vallaeys $^{1}$, (2006), considerado uno de los pioneros en Rsu, enfatiza en la importancia de la toma de conciencia ligada a la voluntad ética de hacer las cosas bien $^{2}$, considera que se debe crear una especie de articulación entre la ética y la eficacia, dando paso a la forma holística de realizar las labores dentro de la universidad en sus funciones de: docencia, investigación, extensión y gestión a través de los indicadores RSU.

El no implementar prácticas de responsabilidad social en las IES a través de sus programas de estudio puede derivar en comportamientos negativos por parte de los egresados, que dañan la convivencia provocando tendencias sociales que afectan a la sociedad como son la corrupción, el egocentrismo, la falta de valores y de conciencia social.

1 El autor hace hincapié en la formación ética para el desarrollo de la RSU.

2 Lo que es bueno hacer o dejar de hacer depende de la negociación entre los afectados y los interesados. 


\section{Marco conceptual}

\section{Responsabilidad Social Universitaria}

Las universidades como sociedades del conocimiento y responsables de marcar la diferencia en cuanto a la productividad y competitividad mediante, el uso de nuevas tecnologías y la automatización de los procesos, les corresponde a las IEs, ser factor clave para el desarrollo del país, esto de acuerdo a lo planteado dentro de la Conferencia Regional de Educación Superior (CRES), 2008 en la cual se marca a la educación superior como un "bien público, e instrumento estratégico de desarrollo sustentable y de cooperación con el cual se busca la conformación de un Espacio Común del Conocimiento y Educación Superior en América Latina y el Caribe" (Valarezo y Túñez, 2014). Otorgando un gran significado a la RSU dentro de las IEs.

Medir la RSU no es tarea fácil, ya que aún no se ha definido claramente el concepto como tal y se siguen incorporando nuevos elementos. Tanto para las IEs como para la comunidad universitaria y, sus autoridades el reto apenas inicia dando un valor relevante a la RSU, ya que estas afrontan múltiples retos bajo la presión de cumplir con una serie de indicadores específicos que les ayuden a conseguir certificaciones de calidad y garanticen mayores ingresos, al mismo tiempo que desarrollan una visión estratégica con políticas claras y transparentes, con liderazgo y talento humano que gestione el conocimiento a lo largo de la vida, "asumiendo su responsabilidad social como una premisa diseñada para construir sociedades inclusivas del conocimiento y desarrollo sustentable para todos los países”, (Aponte, 2008).

Para Martínez Domínguez (2013), la responsabilidad social educativa es "una disposición voluntaria de las instituciones educativas que favorecen el despliegue de sus miembros hacia los demás en un espacio natural con posibilidades sociales".

De acuerdo a Vallaey's (2009), define la responsabilidad social universitaria "como una política de mejora continua de la universidad hacia el cumplimiento efectivo de su misión social mediante cuatro procesos: a) Gestión ética y ambiental de la Institución, b) Formación de ciudadanos responsables y solidarios, c) Producción y difusión de 
conocimientos socialmente pertinentes y d) Participación social en promoción de un desarrollo más humano y sostenible" (Vallaey's 2009).

Para el Proyecto Universidad Construye País de Chile (PUCP), (2006) una universidad es socialmente responsable "por la capacidad prospectiva y transformadora que tiene como organización social, al difundir y poner en práctica un conjunto de principios y valores mediante la gestión inteligente de los impactos: organizacional, ambiental educativo, cognitivo y social, producidos por medio de sus proceso claves: gestión, docencia, investigación y extensión, a través de política éticas de calidad, en diálogo participativo con la sociedad con quien busca en conjunto, un desarrollo humano y sustentable y el cual concreta la demanda ética de justicia y equidad social” (PUCP, 2006).

Mientras que para la Asociación de Universidades Jesuitas de América Latina (2007) es "la habilidad y efectividad de una universidad para responder a las necesidades de transformación de la sociedad donde está inmersa, mediante el ejercicio".

Las diferentes definiciones tienen como elemento en común la percepción que tienen los autores de la gestión para responder a las necesidades de la sociedad y la forma de evaluar los impactos generados por la actividad de las IEs, mediante ciertos indicadores que ayudan a medir o evaluar el cumplimiento de los objetivos propuestos.

Por tanto, el quehacer universitario se encuentra directamente ligado a las necesidades de los diferentes sectores de la sociedad, en cumplimiento a sus funciones de docencia, investigación, extensión y gestión, bajo un marco de comportamiento socialmente responsable, que busca el beneficio de sus grupos de interés para dar respuesta a los requerimientos de la sociedad actual, razones suficientes que justifican la aplicación de la responsabilidad social al funcionamiento de las universidades (Gaete, 2012).

\section{Indicadores de Responsabilidad Social Universitaria}

Cualquier organización que aspire a la competitividad necesita medirse o compararse ya sea en acciones, procesos, procedimientos, prácticas u otros, mediante indicadores. En el caso de la Rsu, se han creado 
instrumentos de gestión que garanticen que las acciones realizadas se encuentran encaminadas al bien común, obteniendo en algunos casos certificaciones o acreditaciones.

Los instrumentos de calidad y prácticas responsables agregan valor a la marca y rentabilidad de la entidad, al mismo tiempo que acredita ante sus empleados, proveedores, clientes, inversionistas que forman parte de los stakeholders "como una organización comprometida de forma voluntaria con una gestión socialmente responsable como parte de su cultura organizacional y estrategia empresarial" (Yfarraguerri, 2014).

Los instrumentos se convierten en indicadores cuando ayudan a medir el logro de objetivos, además de ser un punto de referencia para el seguimiento y avance en los resultados de proyectos y programas. Los indicadores pueden ser cualitativos o cuantitativos, siendo estos últimos los más utilizados sobre todo en cuestiones financieras o de comparación. Cuando se trata de medir la RSU, Yfarraguerri (2014), menciona que se deben cumplir con tres requisitos para ser considerados como indicadores, que son:

1. Fiabilidad. Que ofrezca la misma respuesta si se aplica más de una vez.

2. Comparabilidad. Que se pueda comparar o aplicarse a través del tiempo y en más de una entidad.

3. Validez. Que mida aquello para lo que fue proyectado.

Los principios del Pacto Mundial y el Decálogo de Cemefi, así como los tópicos de Ethos, son los documentos que fincan las bases y marcan el rumbo adaptados a las necesidades de las áreas de la responsabilidad social universitaria. El Manual ${ }^{3}$ de primeros pasos de la Rsu, elaborado por el Banco Mundial de Desarrollo (BID) y la Pontificia Universidad Católica, ha servido como punto de partida por las IES mexicanas y latinoamericanas. La metodología propuesta por Vallaeys, De la Cruz y Sasia (2009), cuyo Manual consta de dos partes,

3 Documento elaborado en 2009 por Francois Vallaeys, Cristina de la Cruz y Pedro M. Sasia. 
una teórica, que permite precisar qué es la Rsu, comprender su legitimidad y urgencia, aclarar dudas, despejar prejuicios y entablar una reflexión institucional participativa en la que todos los actores de la comunidad universitaria comprendan, se convenzan y comprometan con "la universidad que se quiere ser".

La parte práctica es un conjunto de herramientas de autodiagnóstico que evalúan a la universidad en sus diferentes ámbitos, con el fin de conocer cuál es la situación actual respecto a la RSU; establece el alcance en cuatro ámbitos: organizacional, educativo, del conocimiento y social. Se recomienda diseñar el itinerario de los principales pasos en la implementación de la dinámica que responda a la pregunta:

¿Qué tenemos que hacer para mejorar nuestra Rsu?

El manual propone un proceso de cuatro pasos: compromiso, autodiagnóstico, cumplimiento y rendición de cuentas:

1. Compromiso de la dirección en implicar a toda la comunidad universitaria, convencer a los actores a comprometerse con la institución.

2. Autodiagnóstico y análisis mediante herramientas cuantitativas y cualitativas de los cuatro ámbitos clave: formación, conocimiento, investigación y participación social.

3. Cumplimiento al contrastar los resultados del autodiagnóstico con la misión de la universidad y seleccionar las áreas de mejora.

4. Rendición de cuentas, ideas para evaluar y comunicar de forma transparente los resultados de los proyectos, reportar a los grupos de interés.

En la construcción de indicadores de Rsu, Vallaeýs, De la Cruz y Sasia, (2009), consideran que existen cinco componentes centrales que constituyen las metas políticas para el desarrollo de una estrategia integral y sustentable en:

1. Gestión ética y calidad de vida institucional. Promueve la equidad y desecha la discriminación en la comunidad universitaria. 
2. Gestión medioambiental responsable. Fomenta el respeto al medio ambiente con un comportamiento ecológico cotidiano responsable.

3. Participación social responsable. Promueve el desarrollo humano sostenible con un comportamiento solidario de la comunidad universitaria y sus colaboradores.

4. Formación académica socialmente responsable. Que favorezca la responsabilidad ambiental y el desarrollo humano en su formación profesional.

5. Investigación socialmente útil y gestión social del conocimiento. Impulsa el trabajo de investigación, al diagnóstico de necesidades sociales de conocimiento en su entorno y trasmitir los conocimientos interdisciplinarios y congruentes con el desarrollo humano sostenible.

En el Manual de Vallaeys, De la Cruz y Sacia (2009), se considera que los impactos generados por la institución se pueden presentar en cuatro categorías: organizacionales, educativos, cognitivos y sociales:

Impactos organizacionales, que contempla aspectos laborales de los docentes, estudiantes y administrativos, es la forma en que se organizan las tareas que impactan ambientalmente, como los desechos, la desforestación, entre otros, estos miden la huella social y ambiental de la RSU.

Impactos educativos, como la influencia que se tiene en la formación de los jóvenes profesionales, sus valores, la ética profesional y su rol social. Mide el tipo de profesionales, ciudadanos y personas que forma la universidad y la manera en que organiza la enseñanza-aprendizaje.

Impactos cognitivos, orientan la producción del conocimiento, influye en la definición de ciencia, racionalidad, legitimidad, enseñanza. Incentiva y fragmenta los saberes, controla la apropiación social del conocimiento, mide el tipo de conocimientos que se producen en la universidad por su pertenencia social y por sus destinatarios. 
Impactos sociales, este aspecto se tiene un gran peso social, promueve el progreso al crear el capital social, vincular al estudiante con el entorno y sus necesidades, hacer accesible el conocimiento para todos. Mide el acompañamiento en el desarrollo de la sociedad y la ayuda para resolver los problemas fundamentales.

Los ejes transversales que contempla la RSU son los siguientes: a) campus responsable, b) formación profesional ciudadana, c) gestión social del conocimiento y d) participación socialmente responsable. Estos ejes dentro del Manual ayudan a establecer cinco componentes centrales que constituyen las metas políticas para el desarrollo de la estrategia integral sustentable.

Cada componente cuenta con una serie de indicadores, los cuales permiten medir el impacto bajo la condiciónante de que se trata de una propuesta que cada institución adecuará a las necesidades que cada una tenga practicadas en forma democrática, dialógica y consensuada entre los múltiples actores universitarios y sociales Vallaeys (2015). Lo anterior tratando de cubrir en cada uno de ellos los siguientes aspectos:

1. Gestión ética y calidad de vida institucional. Promueve la equidad y desecha la discriminación en la comunidad universitaria.

2. Gestión medioambiental responsable. Fomenta el respeto al medio ambiente con un comportamiento ecológico cotidiano responsable.

3. Participación social responsable. Promueve el desarrollo humano sostenible con un comportamiento solidario de la comunidad universitaria y sus colaboradores.

4. Formación académica socialmente responsable. Que favorezca la responsabilidad ambiental y el desarrollo humano en su formación profesional.

5. Investigación socialmente útil y gestión social del conocimiento. Impulsa el trabajo de investigación, diagnóstico de necesidades sociales de conocimiento requerida por el entorno y la trasmisión de conocimientos interdisciplinarios y congruentes con el desarrollo humano sostenible. 
La experiencia en la Gestión de la RSU Ausjal ${ }^{4}$ reconoce cuatro aspectos clave $e^{5}$ que constituyen el punto de referencia en cuanto a políticas de RSU: a) experiencia vivencial del servicio universitario con las comunidades pobres, b) conocimiento y comprensión causal de la historia y problemas de gobernabilidad, c) alta capacidad técnica y profesional de los estudios, y d) sentido de lo público como espacio de la trascendencia en el quehacer profesional.

Las universidades de Ausjal, ha adoptado dos instrumentos: el primero forma parte del "Sistema de Autoevaluación", y el segundo es un cuestionario propuesto por De la Calle, et al. (2008).

El sistema de Autoevaluación y Gestión de RSU Ausjal, como herramienta de autodiagnóstico, permite conocer el progreso y el comportamiento paso a paso, así como el alcance de las acciones realizadas por las universidades participantes. La estructura de este instrumento se relaciona con cinco dimensiones de impacto: educativo, cognoscitivo y epistemológico, social, organizacional y ambiental (Yfarraguerri, 2014).

De estas dimensiones parte el sistema de información que se compone de dos instrumentos, "un sistema de indicadores institucionales y una encuesta de percepción de los diferentes públicos afectados por la gestión universitaria" A saber:

Indicadores de información institucional. Son indicadores de procedimientos existentes que se miden en porcentajes.

Encuesta de percepción. Proceso que atraviesa cada institución y la apreciación que algunos indicadores o áreas de impacto pueden adquirir para los miembros de la comunidad universitaria.

Lo que busca el sistema de autoevaluación y gestión de la RSU Ausjal es: fomentar el compromiso de toda la institución en la gestión y aplicación de Rsu, visualizar los aspectos relevantes de la situación, facilitar el seguimiento y autodiagnóstico de manera sistemática y periódica, facilitar el análisis comparativo de los procesos, tener insumos

4 Red de homólogos de Responsabilidad Social Universitaria con la participación de 26 universidades o Centros e Institutos de Estudios Superiores en Latinoamérica. Recuperado de http://www.ausjal.org/responsabilidad-social-universitaria.html

5 Aspectos definidos en el I Encuentro de Responsabilidad Social de Ausjal. 
objetivos que faciliten la toma de decisiones y mejorar la gestión institucional de las universidades.

Por su parte, el Proyecto Universidad Construye País de Chile (PUCP, 2006) ${ }^{6}$, nace en 2001 y se expande el concepto y la práctica de RSU, en un conjunto de indicadores esperando que su aplicación contribuya a la difusión del tema dentro de las universidades, proporciona información para tomar acciones concretas y da a conocer el grado de desarrollo de un conjunto de valores y principios que orientan las acciones y el desarrollo humano en gestión, docencia, investigación y extensión de toda la comunidad universitaria.

Para el PUPC, en concordancia con la Unesco de acuerdo a la Conferencia Mundial sobre Educación Superior realizada en París (1998) y los maestros participantes, se visualizan como universidad socialmente responsable los siguientes aspectos:

f. Preservar y crear el capital del saber y del pensamiento, mediante la reflexión y la investigación interdisciplinaria difundida por diferentes medios.

g. Formación de hombres y mujeres altamente calificadas, integrales y comprometidas con valores.

h. Incluir en el currículo una visión de la realidad del país.

i. Ofrecer formación permanente y facilitar el reingreso a las IES para la actualización permanente.

j. Abrirse al cambio valorando e incorporando conocimientos y experiencias del entorno.

Para precisar mejor el concepto de responsabilidad social universitaria, en el proyecto UCP se deben contestar las siguientes preguntas:

¿De qué es responsable la universidad?, ¿ante quién es responsable? y ¿cómo es responsable?

Los stakeholders son definidos por Vallaeys, De la Cruz y Sasia (2009) como partes interesadas a "cualquier individuo o grupo que

6 Iniciativa impulsada por la Corporación Participa y la Fundación Avina a la cual se unen 11 universidades chilenas, inspirada en valores humanistas. 
pueda afectar o ser afectado por las políticas, objetivos, decisiones y acciones de la universidad". En el caso de la Rsu, está integrado por docentes, investigadores, autoridades, estudiantes, proveedores, egresados, competidores, comunidades locales, organizaciones sociales y gobierno.

Los mismos autores definen la buena gobernabilidad, que se presenta cuando la organización define su misión y se entrega a ella: implementa un código de ética y un comité encargado de promoverlo que asegure un buen clima laboral; que lucha contra la discriminación y desigualdad; proteja los derechos de las personas y se comprometa a rendir cuentas. Estas son parte de las buenas prácticas que ayudarán a evitar el riesgo de corrupción en la organización (Vallaeys, De la Cruz y Sasia, (2009).

\section{Resultados}

El instrumento se construyó considerando los tres modelos ya mencionados (Vallaeys, Ausjal y PUCP), ya que entre ellos se repiten ciertos indicadores, pero la principal fuente para su desarrollo fue el Manual de buenas prácticas, debido en parte a que el modelo es adaptable en forma más general a cualquier institución, haciendo las adecuaciones necesarias.

El modelo de Ausjal fue desarrollado por universidades jesuitas, es un modelo que utiliza indicadores enfocados a aspectos más espirituales, maneja muchos valores y ética, dando menos peso al aspecto cognitivo y cuidado del medio ambiente. Mientras que el PUCP, al igual que Ausjal está enfocado a una red de universidades, más que al uso individual, también mide aspectos espirituales, pero está considerado funcionar más bien como una red. Todos los modelos se enfocan en la medición del impacto y el diagnóstico como punto de partida para la implementación de la Rsu.

El instrumento utilizado en la investigación está basado mayormente en el modelo expuesto por Vallaeys, De la Cruz y Sasia, (2009). Se optó por elaborar tres diferentes tipos de encuestas una para docentes, otra para alumnos y la última para el personal administrativo. Cada una midiendo diferentes indicadores, considerando más extensa la encuesta de los docentes porque contempla la parte de investigación o gestión del conocimiento, cuyos resultados se presentarán en la siguiente investigación. 


\section{Conclusiones}

Gestionar la universidad con criterios de RSU no es responder a leyes económicas del mercado, donde se considera que el alumno asume el papel del cliente y la educación superior como el producto, esto va más allá, se trata de medir el impacto de las acciones que realizan las universidades para resolver la problemática actual y los nuevos desafíos que se presentan. Uno de los desafíos de la RSU será promover nuevas formas de creación y trasmisión de conocimiento; flexibilidad en sus planes de estudio; incorporar nuevos elementos en el currículo; diversificación de la demanda por la aparición de nuevas modalidades e instituciones en la transferencia del conocimiento y mayor uso de las tecnologías de información y comunicación, pero con ética y valores, donde el alumno se encargue de su aprendizaje para toda la vida.

Algunas universidades, como es el caso de Chile, ya se encuentran inmersas en esta dinámica de la RSU aún sin integrar a todos los elementos como una política de gestión universitaria y filosofía de vida. Otros países, como México, recién empiezan a incursionar en este nuevo desafío, sin embargo, algunas universidades ya dieron los primeros pasos. Como se ha podido constatar en los modelos, la Rsu se encuentra vinculada a los impactos que generan las IEs, en sus prácticas, principios y valores éticos mediante los procesos de gestión, docencia, investigación y extensión.

En el caso de la Facultad de Contaduría y Administración se elaboró un instrumento propio y se encuentra en el proceso de aplicación a efectos de iniciar con el diagnóstico. Al mismo tiempo ya se han establecido lineamientos dentro del Plan de Desarrollo Institucional (2015-2019), incluyendo aspectos como el cuidado al medio ambiente, la equidad de género y educación con valores.

A fin de iniciar la marcha hacia una universidad socialmente responsable presentarán posteriormente los resultados de la aplicación del instrumento. Si bien es cierto que los indicadores facilitan la medición de las variables, el diagnóstico es sumamente necesario como punto de partida y para conocer la situación actual de la institución. 


\section{Referencias}

Aponte, E. (2008). Tendencias de la Educación Superior en América Latina y el Caribe. En A.L. Gazzola y A. Drikisson (Eds.), Desigualdad, inclusión y equidad en la educación superior en América Latina y el Caribe: Tendencias y escenarios alternativos en el horizonte 2021. Asociación Colombiana de Universidades - Caracas Ministerio de Educación Superior. Recuperado de http://unescoeducacionsuperior.uprrp.edu/wp-content/ uploads/2011/11/Tendencias-de-la-Educaci\% C3\%B3n-Superior-enAmerica-Latina-y-elCaribe.pdf

De la Calle, M. (2008). La formación de la Responsabilidad Social del Universitario: un estudio empírico. Madrid: Universidad Complutense de Madrid.

Domínguez, M. (2009). Responsabilidad Social Universitaria. Humanismo y Trabajo Social. Issn 1696-7623. Recuperado de http://www4.unileon. es/trabajo_social/presentacion.asp

Gaete Quezada (2012). Responsabilidad social universitaria: una nueva mirada a la relación de la universidad con la sociedad desde la perspectiva de las partes interesadas. Un estudio de caso. (Tesis doctoral). Valladolid, España: Facultad de Educación y Trabajo Social de la Universidad de Valladolid.

Hernández, R., y Saldarriaga, A., (2009). Gestión de la Responsabilidad Social Universitaria. Dyna, Año 76, N. ${ }^{\circ}$ 159. Medellín, Colombia. IssN. 0012-7353.

Martínez de Carrasquero, C. (Enero, 2011). Responsabilidad social universitaria y su articulación con las Funciones docencia-investigación-extensión para su vinculación con el Entorno Social. IESALC, Informe de Educación Superior. Boletín N. ${ }^{\circ} 214$.

Martínez Domínguez, L. (2013). Responsabilidad social y comunicación institucional en los centros educativos. Estudios sobre educación, 27, 169191. DOI 10.15581/004.27.169-191

Plan de Desarrollo Institucional (PDI). Universidad Autónoma de Baja California, UABC, (2015-2019), (pp.43-50). Recuperado de http://www.uabc. $\mathrm{mx} /$ planeacion/pdi/2015-2019/

Red de RSU Ausjal (2009). Politicas y sistema de autoevaluación y gestión de la responsabilidad social universitaria en Ausjal. Córdoba, Argentina: Editorial Alejandría. 
Universidad Construye País (2006). Responsabilidad social universitaria: una manera de ser universidad. Teoría y práctica en la experiencia chilena $\left(1^{\mathrm{a}}\right.$. ed.). Santiago de Chile. ISBN 956-8140-08-5.

Universidad Construye País (2004). Observando la responsabilidad social universitaria. Documento de trabajo (versión actualizada). Santiago de Chile: Proyecto Universidad Construye País.

Valarezo González, K., (2011). Responsabilidad Social Universitaria: Mapa del área andina y estrategias de comunicación empleadas para su difusión. Universidad de Santiago Compostela.

Valarezo, K. y Túñez, J.M. (2014). Responsabilidad social universitaria. Apuntes para un modelo de Rsu. Revista de Comunicación, 13, 84-117.

Vallaeys, F. (2006). Breve marco teórico de la Responsabilidad social universitaria; Recuperado de hht://rsuniversitaria.org/web/imágenes/stories/ BreveMarcoTeodelaResponsabilidadSocialUniv.pdf

Vallaeys, F., De la Cruz y Sasia P., (2009). Responsabilidad social universitaria. Manual de primeros pasos. McGraw-Hill y Banco Interamericano de Desarrollo (BID). Recuperado de https://publications.iadb.org/bitstream/handle/11319/245/Responsabilidad\%20social\%20universitaria. pdf? sequence $=1 \&$ isAllowed $=y$

Vallaeys F., (2009). "Responsabilidad Social Universitaria: una nueva filosofía de gestión ética e inteligente para las universidades". Revista educación superior y sociedad: nueva época, 13(2), 193-220.

Vallaeys F., (2014). Hacia la construcción de indicadores de Responsabilidad Social Universitaria. Lima Perú.

Vázquez, S., Ponce, G., Cervantes, P., Meléndez, E., y Piñón, V. (2006). Vinculación universidad- empresa-Estado en la realidad actual de la industria farmacéutica Mexicana. Congreso Retos y expectativas de la Universidad.

Yfarraguerri L., (2014). Necesidad de indicadores de medición de la responsabilidad social en las universidades. Daena Internatinal Journal of Good Conscience, 9(1), 142-157. IssN 1870-557X. 